\title{
LATINOAMÉRICA Y DESARROLLO, PERSPECTIVAS Y PROGRESOS A TRAVÉS DE ARTÍCULOS EN REVISTAS GEOGRÁFICAS ALEMANAS Y ESTADOUNIDENSES (1980-1994)
}

\author{
POR \\ M. ${ }^{a}$ ASUNCIÓN MARTÍN LOU \\ $\mathrm{Y}$
}

MERTEN SIEVERS

\section{Introducción}

Este artículo pretende dar una noción de lo que ha sido en los últimos años el estudio de la problemática del desarrollo en los países Latinoamericanos dentro de la Geografía alemana y estadounidense, a través de un estudio bibliográfico sobre este tema realizado en revistas geográficas de estos países y publicadas en los últimos quince años*.

En el estudio se ha revisado un total de ocho revistas desde 1980 a 1994, cinco alemanas (las tres primeras de la ex RFA y las otras dos de la ex RDA): Die Erde, Erdkunde, Geographische Zeitschrift, Geographische Berichte y Petermanns Geographische Mitteilungen, y tres

\footnotetext{
* El estudio se ha realizado como actividad investigadora del Programa Erasmus por Merten Sievers, bajo la dirección de M. ${ }^{a}$ A. Martín Lou, en el Instituto de Economía y Geografía del CSIC, Madrid.
} 
estadounidenses: Annals of the Association of American Geographers, Geographical Review y The Professional Geographer.

Se han elegido estas ocho revistas, pertenecientes al fondo bibliográfico de la biblioteca del I.E.G., dada la importancia científica de las mismas y su amplia difusión dentro del ámbito de la Geografía. Las revistas analizadas pretenden difundir los conocimientos geográficos, así como su evolución. Un estudio de sus contenidos puede revelar tendencias, perspectivas, formas y avances de lo que ha sido el estudio del desarrollo en Latinoamérica en los últimos quince años.

$\mathrm{Al}$ realizar este análisis se nos plantean varias preguntas:

¿Cuáles son los temas que principalmente se ha tratado y cómo han evolucionado en los últimos quince años? ¿Han aparecido temas nuevos? ¿Han desaparecido otros?

¿Qué se escribe sobre Latinoamérica y cuál es la percepción de los autores sobre este continente? ¿Se escribe prioritariamente sobre ciertos temas y sobre ciertas áreas y espacios dentro de Latinoamérica? ¿Cuál es la distribución geográfica de los artículos? ¿Qué ideas de desarrollo tienen los autores? ¿Hay diferentes corrientes en cuanto al pensamiento sobre el desarrollo, cómo evolucionan las ideas de desarrollo y qué causas del desarrollo o del «subdesarrollo» se mencionan y cuáles son las propuestas para un desarrollo futuro? ¿Se relaciona el desarrollo con el espacio y su estructura? ¿En qué forma?

¿Se utilizan diferentes modelos y conceptos geográficos? Y si se utilizan, ¿cuáles son? ¿Se puede considerar que todos los textos son geográficos? ¿Se introducen nuevos modelos, conceptos o teorías? ¿A qué escala trabajan los autores? ¿Son principalmente locales, regionales, nacionales?, ¿cómo conectan las tres escalas? Y, finalmente, ¿se detecta un progreso geográfico en esta línea de investigación?

En fin, se trata de preguntar si la Geografía puede hacer proposiciones válidas para el desarrollo dentro de Latinoamérica $\mathrm{y}$, suponiendo que sí, cuáles son. $\mathrm{O}$ si la Geografía sólo ha aportado la mera descripción de lugares exóticos: «Desafortunadamente, el mayor interés de muchos practicantes de nuestra disciplina ha sido, y sigue siendo, descripciones objetivas de lugares exóticos» (Riddell, 1989). 
Importante o más bien crucial en este contexto resulta preguntar: ¿qué es desarrollo? Sobre este tema se han escrito muchísimos libros, por lo cual no es posible responder adecuadamente, ni mucho menos, exhaustivamente a esta pregunta, sirva la cuestión sólo para hacer algunos comentarios.

Rechazamos una visión del desarrollo puramente economista, es decir, la que considera exitoso el desarrollo si logra subir los niveles medios de renta per cápita. Aunque la mejora de la base material es una parte importante de lo que se podría llamar desarrollo, ésta no puede ser medida sólo con parámetros como el nivel de renta o el PNB.

Por tanto, lo que hay que preguntar acerca del desarrollo de un país es: «qué ha sucedido con la pobreza, con el desempleo y con la desigualdad» (D. Seer citado en Todaro, 1985). Además de parámetros económicos hay otros factores sociales, culturales y políticos importantes dentro del desarrollo. Al igual que los parámetros económicos éstos otros dependen de ciertos principios de valor, éticos o normativos: «Conceptos y objetivos tales como igualdad económica y social, erradicación de la pobreza, educación para todos, mejora de nivel de vida, independencia nacional, modernización de las instituciones, participación política y económica, democracia, no dependencia y satisfacción personal se derivan, todos ellos, de juicios de valor subjetivos sobre lo que es bueno y deseable y lo que no lo es» (Todaro, 1985).

Sabiendo esto, también habría que preguntar si estos sistemas y leyes son universales, o si más bien los conceptos occidentales normalmente utilizados tienen poca o reducida validez en otros espacios sociales y culturales.

Además de estas preguntas sobre lo que es desarrollo, habría que plantearse otras también estrechamente relacionadas con el tema: «... La cuestión no es en qué consiste el desarrollo y cómo debería medirse y promoverse. Es más una cuestión de quién lo dice en qué términos y para quién. Quién está hablando, para quién y sobre quién recaen las miradas del promotor y con qué efecto» (Corbridge, 1992).

Aunque seamos conscientes de qué valores y legislaciones pueden diferir en diferentes épocas y diferentes espacios sociales y culturales, siempre hay que tener en cuenta que la mejora de condicio- 
nes económicas, sociales, culturales y políticas denominadas de «desarrollo» son una meta y un problema global, y que «el desarrollo y el subdesarrollo son aspectos diferentes del mismo proceso universal» (Blomstrom, Hettne, 1990). Lo cual nos lleva a la pregunta (o a la conclusión) de si el desarrollo de una parte del mundo condiciona el desarrollo o «subdesarrollo» de la otra parte, y de nuevo nos planteamos la duda del desarrollo ¿para quién y en beneficio de quién?

El artículo se divide en cuatro partes, en las cuales se tratará de hallar respuesta a las cuestiones planteadas, de mostrarnos cómo han evolucionado estos temas en las revistas en los últimos quince años y plantearnos también alguna que otra pregunta crítica. Primero se da una idea general de lo que se ha escrito sobre Latinoamérica en las revistas, para después examinar los artículos de las revistas alemanas que tengan una conexión con el desarrollo, por períodos de cinco años. $Y$ de igual manera actuaremos con los artículos aparecidos en las revistas estadounidenses. En la tercera parte se compara los artículos de ambos países, en cuanto a sus similitudes y diferencias y se enmarcan las tendencias futuras de ambos espacios. En la última parte se plantea la pregunta de si ha habido un progreso en la evolución de los temas dentro de la Geografía, concluyendo con una crítica personal de los artículos.

\section{Articulos sobre Latinoamérica en las revistas alemanes}

El contenido de las revistas, lógicamente, es muy heterogéneo y cambiante tanto cuantitativa como cualitativamente. Lo es también el número y el contenido de los artículos que aparecen en ellas sobre Latinoamérica. Mientras que en la revista Erdkunde se han publicado cuarenta y dos artículos en estos quince años, en Petermanns Geographische Mitteilungen sólo han sido cinco en total. El peso que da cada una de las revistas dedicadas a temas de Geografía Humana y Física sobre Latinoamérica se refleja en el cuadro I.

Aunque haya un mayor número de artículos en las revistas alemanas, también es mayor la proporción de artículos de Geografía Física, por lo cual, los artículos donde potencialmente podrían (o debe-

$$
-96-
$$


Cuadro I

\begin{tabular}{lccr} 
Revista & Geo. Humana & Geo. Física & Total \\
\hline Die Erde & 11 & 8 & 19 \\
Erdkunde & 19 & 23 & 42 \\
Geogr. Zeitschrift & 28 & 1 & 29 \\
Geogr. Berichte & 5 & 0 & 5 \\
Petermanns G. M. & 3 & 2 & 5 \\
Annals of the AAG & 13 & 2 & 15 \\
Geographical Review & 37 & 3 & 40 \\
Porf. Geographer & 10 & 2 & 12 \\
TOTALES & 126 & 41 & 167
\end{tabular}

rían) aparecer aspectos vinculados al desarrollo viene a ser casi el mismo. Cabe destacar que en los dos países sobresale una revista por cada uno de ellos en la que se detecta gran número de artículos dedicados a Latinoamérica dentro de la la línea de Geografía Humana: Geographische Zeitschrift (28) y Geographical Review (37).

Para dar una idea más clara de la evolución de estos temas en las revistas se dividen los quince años del análisis en tres períodos de cinco años (cuadros II y III).

CuAdro II

NÚMERO DE ARTÍCULOS PUBLICADOS EN LAS REVISTAS ALEMANAS, 1980-1994

\begin{tabular}{ccccccccc} 
Períodos & Fis. & Urb. & Econ. & Agr. & Hist. & Otr. & Rel. Des. Total \\
\hline $1980-1984$ & 9 & 5 & 5 & 4 & 0 & 6 & 15 & 29 \\
$1985-1989$ & 8 & 6 & 9 & 5 & 5 & 3 & 22 & 36 \\
1990-1994 & 17 & 6 & 6 & 1 & 2 & 3 & 14 & 35 \\
TOTAL & 34 & 17 & 20 & 10 & 7 & 12 & 51 & 100
\end{tabular}

El porcentaje de estos artículos dedicados a los diferentes países es: México, 24 \%; Brasil, 10 \%; Bolivia, $10 \%$; Perú, 6 \%; Ecuador, 6 \%; resto de países, $44 \%$.

El porcentaje de estos artículos dedicados a los diferentes países es: Brasil, 18 \%; México, 16,5 \%; Perú, 15 \%; República Dominicana, 4,5 \%; Costa Rica, 4,5 \%; restos de países, 41,5\%. 


\begin{tabular}{ccccccrrr}
\hline \multicolumn{8}{c}{ CUADRo III } \\
\multicolumn{10}{c}{ NÚMERO DE ARTÍCULOS PUBLICADOS EN LAS REVISTAS } \\
ESTADOUNIDENSES, 1980-1994 \\
Períodos & Fis. & Urb. & Econ. & Agr. & Hist. & Otr. & Rel. Des. Total \\
\hline $1980-1984$ & 2 & 3 & 5 & 5 & 7 & 4 & 13 & 26 \\
$1985-1989$ & 1 & 0 & 4 & 2 & 1 & 1 & 8 & 9 \\
$1990-1994$ & 4 & 3 & 4 & 6 & 7 & 8 & 12 & 12 \\
TOTAL & 7 & 6 & 13 & 13 & 15 & 15 & 33 & 67
\end{tabular}

Dentro de las revistas alemanas, 51 de 66 artículos de Geografía Humana sobre Latinoamérica mencionan de alguna manera la problemática del desarrollo, mientras que de los 60 artículos de esta misma materia en las revistas estadounidenses sólo 33 tienen que ver con el desarrollo. Esto se debe, en parte, a que muchos artículos de las revistas de Estados Unidos son de Geografía Histórica coincidiendo con la conmemoración del Descubrimiento Colombino y en éstos raramente se hace referencia al desarrollo. Pero, en parte, también puede deberse a que algunos autores conciben el desarrollo como un proceso histórico casi automático, lo que en algunos casos harían innecesaria su mención.

En ambos países hay cierta tendencia a una concentración espacial en cuanto a los territorios y países analizados. Los países más estudiados son México y Brasil, seguidos por los Países Andinos en el caso alemán y por Costa Rica y la República Dominicana en el caso estadounidense.

Las razones de esta atención espacial podría ser, en parte, la importancia económica y demográfica de estos países y, en parte, la existencia en ellos de culturas tradicionalmente estudiadas. Pero éstos no pueden ser los únicos factores que explican la distribución espacial. Por ejemplo, apenas aparecen Colombia o Argentina u otros países económica y demográficamente más importantes que Bolivia o Costa Rica, cuyos territorios no son objeto de estudio en la serie analizada. Cabe pensar también que, como el número de autores que publican en estas revistas sobre Latinoamérica no es muy grande, las prioridades personales adquieren un peso significativo en la distribución espacial. 
Referencias en las revistas alemanas, 1980-1994.

Revisaremos ahora los artículos de las revistas alemanas por espacios de cinco años, para al final hacer un comentario sobre la evolución de esta temática. Se analizan principalmente los textos de las revistas de la ex RFA, ya que el número de artículos en revistas de la ex RDA es muy reducido. De estas últimas, Geographische Berichte se extinguió en 1990 y Petermans Geographisches Mitteilungen cambió tan radicalmente de política que guarda poca relación con lo que era antes de la reunificación alemana.

En el primer período analizado, 1980 a 1984, se encuentran sobre todo textos relacionados con la estructura agraria y el desarrollo rural (Sander, 1981; Mahnke, 1982; Friebel, 1983; Schacht, 1980; Roenick, 1983), textos relacionados con estructuras urbanas, problemas de vivienda urbana y modelos urbanos (Gormsen, 1981; Nuhn, 1981; Baehr-Mertins, 1981; Brogner, 1984; Buchhofer, 1984) y con otros temas (Gormsen, 1983; Mikes, 1982; Bleckert, 1983).

El artículo de Sander analiza las relaciones del turismo con la artesanía y la estructura agraria en la altiplanicie mexicana, y plantea la pregunta si la actividad agraria está en decadencia debido a la expansión de la producción artesanal dedicada al turismo. Después de un largo análisis descriptivo no ofrece una respuesta. Mahnke describe la agricultura indígena en Bolivia en un tono paternalista, criticando las influencias externas y realzando el equilibrio ecológico en el que se encuentra. Friebel analiza la estructura agraria de las comunidades indígenas en la Araucania. Schacht y Roenick analizan proyectos de desarrollo agrario en el noreste brasileño. El primero hace referencia al proyecto de una cooperativa rural iniciado por agencias de desarrollo occidentales y continuado por el programa estatal brasileño Polo-nordeste, llegando a la conclusión de que el proyecto tuvo cierto éxito, pero sin relacionarlo con otros proyectos similares. El artículo de Roenick estudia el programa de desarrollo Polo-nordeste, y menciona conceptos como el desarrollo local y la participación popular pero, aun así, su análisis es muy descriptivo. Gormsen plantea un modelo espacio-temporal de ciudades en la América española, tomando como ejemplo Puebla (México). Distingue tres fases de desarrollo muy ligadas al ejemplo europeo. Concibe la América españo-

$$
-99-
$$


la como un espacio homogéneo y proyecta ciertas características típicamente mexicanas (por ejemplo, las «vecindades») al resto de la región. El artículo de Nuhn es un análisis cuantitativo del sistema de ciudades de América Central y su significado para el desarrollo regional. Nuhn utiliza el término «repúblicas bananeras», lo que permite especular sobre su percepción de estos países. Baehr y Mertins crean un «esquema ideal» para la diferenciación de espacios sociales en ciudades latinoamericanas. Los autores describen los procesos causales de las estructuras espaciales existentes e indican posibles tendencias futuras, en lo que es un modelo bien logrado y accesible que refleja el conocimiento que los autores tienen del contexto latinoamericano. Buchhofer analiza la sociotopografía de una ciudad compañía en México, estudiando las relaciones entre movilidad espacial y la distribución y estabilidad de las rentas. Brogner diferencia la metropolonización en el Primer Mundo y el Tercer Mundo a través de un análisis cuantitativo, resaltando las diferencias en cuanto a crecimiento demográfico y la primacía de las grandes ciudades. Su análisis es muy cuantitativo $\mathrm{y}$, tal vez por eso, algo superficial.

Los tres últimos textos tienen diferentes temáticas. El fenómeno del turismo y su frente pionero en el Tercer Mundo es analizado por Gormsen, tomando como ejemplo a México. Por frente pionero del turismo, Gormsen entiende el avance de una civilización de tipo occidental y altamente tecnolizada sobre áreas de baja densidad de población en el así llamado «Tercer Mundo». Gormsen propone un modelo de tres fases y critica la estrategia oficial de polos de crecimiento turístico, relacionando la problemática local con el ámbito internacional por el concepto centro-periferia y refiriéndose a la dependencia y explotación que sufre el espacio local del frente pionero. El texto de Mikes analiza el mercado de trabajo peruano en conexión con la satisfacción de las necesidades básicas, critica el «desarrollo desde arriba» y da pautas para la planificación a nivel nacional.

El artículo de Bleckert es el único de la ex RDA en este período; describe la relación del Bloque Andino, como él lo llama, con el capital monopolista imperialista, siendo muy superficial y más políticoeconómico que geográfico. 
Así, pues, este período se caracteriza por textos muy descriptivos de los cuales pocos llegan más allá del análisis y apenas se plantean diagnósticos o propuestas. La mayoría de los textos se desarrollan a escala local y muchas veces se echa de menos alguna referencia o conexión con las escalas de nivel nacional o global. También sería de mucha utilidad si los autores se atrevieran a comparar ciertos espacios, para responder a la pregunta: ¿Qué diferencia hay?

En cuanto a la problemática del desarrollo muy pocos autores manejan teorías o conceptos y pocos analizan las causas del desarrollo o subdesarrollo conectando sus explicaciones con teorías más generales. Varios autores hacen ciertas críticas en cuanto al desarrollo existente, esto casi siempre a nivel local, criticando exclusivamente causas endógenas de éste, y proporcionando así sólo explicaciones parciales o erróneas sobre lo que es el desarrollo del espacio en cuestión.

En el segundo periodo, 1985 a 1989, los principales temas son, la planificación y los problemas urbanos (Buchhofer, 1986; HeinebergSchaefers, 1989; Popp, 1985; Baehr-Mertins, 1985; Cotic-Zothner, 1988; Sagawe, 1989), la estructura y producción agraria (Seele, 1986; Rehker, 1989; Hoensch, 1986; Luecker, 1986; Korpe, 1986; Kohlhepp, 1989). En este período tienen un peso mayor los temas referentes a «frente pioneros» (Coy, 1986; Buchhofer, 1988, y también Kohlhepp, 1989) y al turismo (Jurczek, 1985; Ungefehr, 1988; Tyrakowski, 1986).

El artículo de Buchhofer sobre políticas de suelos y construcción de viviendas en dos nuevas ciudades industriales de México compara a ambas y critica las causas endógenas de un desarrollo desequilibrado y con una mala planificación. Pero su análisis no sobrepasa la escala local.

También el texto de Heineberg-Schaefers trata de la planificación urbana en México. Se estudia la metropolinización de Guadalajara. Las conclusiones son que existe una estructura social dualista y graves problemas medioambientales a nivel local. Popp describe la forma y estructura de los «fraccionamientos» (conversión de suelo no urbano a urbano) en Puebla (México), resaltando el control que tienen las élites económicas locales sobre la estructura urbana y la urbanización.

Baehr-Mertins estudia la evolución de la población en Santiago de Chile y da un buen análisis de la situación, apuntando problemas 
existentes y posibles en un futuro cercano. Adolece de alguna referencia clara a la situación política de Chile en ese momento, imprescindible para explicar ciertas estructuras.

El artículo de Sagawe introduce la problemática de la drogadicción y el narcotráfico como cuestión de la Geografía urbana, siendo la problemática social el principal eje del artículo sobre Santo Domingo (República Dominicana). Analiza las causas y efectos a escala local, nacional e internacional y demuestra (aparentemente) un buen conocimiento de la ciudad y sus problemas.

Rehker, Luecker y Korpe tratan la problemática agraria en el contexto brasileño. Rehker estudia la producción de alimentos básicos en el noreste y da un diagnóstico de la situación y una propuesta para el futuro a un nivel local. Luecker describe el cambio estructural agrario en una zona periférica, por políticas orientadas hacia el mercado mundial. El autor considera como periférica una zona situada en el sur brasileño (una de las regiones más desarrolladas del continente) y considera que la política seguida es positiva para el área estudiada, pero no llega a decir nada sobre la escala nacional y las implicaciones de una política orientada al mercado mundial.

El artículo de Korpe estudia cooperativas agrarias en el estado de Paraná y expone de forma descriptiva las causas y efectos del desarrollo económico.

Los artículos sobre la «frontera pionera» se localizan en la Amazonia y el avance de la sociedad de tipo occidental en esta zona en Ecuador y Brasil.

Coy critica el «development from above» practicado por el gobierno brasileño en su estudio sobre el estado de Rondônia. Describe las estructuras espaciales resultantes de la migración regulada y espontánea, resultante a su vez del programa de desarrollo Polo-nordeste. Este programa pretendía un desarrollo rural integrado, pero según el autor se convirtió en una simple ayuda infraestructural.

Buchhofer hace propuestas para mejorar el adecuamiento social, ecológico y de identidad cultural dentro de cooperativas en el «frente pionero» del estado de Paraná. 
El desarrollo urbano en un «frente pionero» es la temática del artículo de Kohlhepp. Analiza las pequeñas ciudades que se han desarrollado muy ligadas a la explotación petrolífera de la Amazonia ecuatoriana. Aunque su análisis a nivel local es concluyente, falta la relación con escalas mayores, interesante en este caso, porque la mayoría de las empresas petrolíferas y gran parte de sus empleados son extranjeros.

Jurczek analiza los impactos socioeconómicos, sobre la estructura social y cultural, del turismo en Perú y Cuzco. Es un análisis a varias escalas, bien estructurado y crítico; llega a la conclusión de que la mayoría de los impactos son negativos o destructivos y el autor recomienda medidas para el futuro.

Ungefehr estudia un proyecto de desarrollo turístico en las Bahamas en relación con la política interior. El artículo es, sobre todo, una crítica a la política bahamiana y una defensa de la libre empresa, desde un punto de vista muy occidental. Tyrakowski hace una descripción folklorística del turismo nacional mexicano.

Un artículo interesante que aparece en este período, pero que es difícil enmarcar en una de las anteriores categorías, es el de Gormsen (Gormsen, 1986). Analiza la producción de flores en la altiplanicie de Bogotá y presenta al aeropuerto de esta ciudad como factor locacional de esta producción. En un estudio muy detallado y fundado teóricamente, el autor analiza las causas y efectos de la producción a escala local e internacional y discute su impacto socioeconómico y regional. Demuestra un buen conocimiento de la situación y da las pautas para un desarrollo futuro y sus problemas.

Los artículos de revistas de la ex RDA en este período (Bleckert, 1986; Waehnelt, 1986; Hoensch, 1986; Cotic-Zothner, 1988) están algo alejados de la realidad latinoamericana, tal vez exceptuando a Cuba, donde todo se presenta de color de rosa. Los autores tienen una idea del desarrollo muy economicista, siendo importante el tema de la inserción de Latinoamérica en el mercado capitalista mundial, en el que se percibe a Latinoamérica como un espacio casi homogéneo.

En este período se aprecia cierto progreso comparándolo con el anterior, aunque, la crítica hecha al último período, en gran parte también es válida para éste. La variedad de los temas tratados es

$$
-103-
$$


mayor y hay más autores que los relacionan a diferentes escalas. Un problema que sigue existiendo es el que muchos autores no consideran necesario mencionar ni siquiera los niveles socioeconómicos de la población.

En cuanto a los textos sobre las fronteras pioneras, un tema que gana peso en este período, en su mayoría mencionan los problemas medioambientales sólo como marginales y nos parece alarmante la no mención de los pueblos indígenas que viven detrás de gran parte de estas «fronteras» y que son los más afectados por su avance.

En el tercer período, 1990 a 1994, los temas son más variados que en los dos anteriores, todavía se puede hablar de artículos relacionados con la planificación y el desarrollo urbano (Schneider-Sliwa, 1991; Gans, 1990; Coy, 1990; Buchhofer-Aguilar, 1991; Sagawe, 1992; Wehrhahn, 1993), con la estructura agraria y la población rural (Breuler, 1990; Wachter, 1992; Birk, 1993), con el desarrollo regional (Gierhake, 1993; Gristai-Treivish, 1990; Gierhake, 1991) y de nuevo con el «frente pionero» (Coy, 1990) y el turismo (Tyrakowski, 1994).

Kingston y la planificación urbana en Jamaica son el tema del artículo de Schneider-Sliwa, que introduce los conceptos de planificación exógena y endógena. Exógena llama a la planificación orientada por ideas occidentales y generadas por fuerzas externas, mientras que endógena llama a la desarrollada con conceptos locales y ejecutadas con medios locales. En el estudio se comparan dos proyectos de revitalización urbana en Kingston, uno de planificación exógenea y otro endógeno. La autora critica los conceptos occidentales, pero también advierte que el desarrollo endógeno no es la «medicina milagrosa» como algunos autores sugieren. Analiza bien la situación y los problemas en los dos casos, pero no llega a hacer propuestas para el futuro.

Gans analiza el desarrollo urbano y el sector informal en Montevideo en uno de los pocos artículos que tratan esta temática. Su análisis es algo paternalista y no llega más allá de una crítica a la situación interna de Uruguay.

Buchhofer-Aguilar estudian la expansión urbana durante la crisis de la deuda en México y concluyen que la movilidad de la clase media en ese período es mayor que la de la alta y la baja, las dos afecta- 
das, de diferente manera, por la crisis. En otro artículo sobre Santo Domingo, Sagawe investiga la conexión existente entre «caudillismo» y desarrollo urbano. Es un buen análisis temporal a escala local y sus implicaciones a escala nacional, aunque después del análisis se hecha de menos un diagnóstico y una propuesta.

Wachter estudia la importancia de la posesión del «título de tierra» para la utilización agraria sostenible y ecológicamente viable en Honduras. Analiza un programa de titulación de tierras del gobierno hondureño, llegando a la conclusión de que esta titulación tiene pocos efectos en cuanto a las cuestiones planteadas.

Birk ofrece un artículo de rasgos sociológicos en el que describe la organización interna y la lógica de los campesinos en las tierras altas de Guatemala. Plantea cuestiones a las cuales, según él, los geógrafos no han prestado bastante atención y con las que se lograría un mejor entendimiento de algunos procesos de la economía local. Es un texto interesante por su temática nueva, y sin duda relevante, pero a veces resulta tener un tono paternalista hacia la población local y tal vez habría que preguntarse si esto es, ahora, Geografía.

Las inversiones públicas en el desarrollo regional boliviano son el tema de Gierhake (1991), que analiza la distribución espacial de las inversiones y su procedencia y pronostica problemas futuros en forma clara y accesible, aunque descriptiva. En su artículo de 1993, Gierhake estudia las estrategias de corporaciones de desarrollo en regiones marginales de tres países andinos: Bolivia, Colombia y Perú.

Si bien representan cierta base para el futuro, estas organizaciones estatales no han podido ofrecer conceptos capaces de convencer a los gobiernos para lograr una mayor asignación de recursos que la actual, que es ínfima. Por ello, también es mínimo el efecto de estas corporaciones sobre el desarrollo regional. El autor demuestra tener buenos conocimientos de las bases legales y hace un análisis y un diagnóstico de la situación, refiriéndose a conceptos como el autodesarrollo y el desarrollo local, orientado al mercado mundial.

Uno de los pocos textos teóricos sobre desarrollo es el de GristaiTreivish que presenta un modelo de etapas de desarrollo, conectándolo con el concepto centro-periferia, basándose en las ondas de Ko-

$$
-105-
$$


drjatieff. Si bien la discusión teórica dentro de la Geografía es importante aunque descuidada, sobre todo en cuanto a teorías de desarrollo, un modelo basado en etapas históricas que tienen su origen en la industrialización europea y norteamericana, es, por lo menos, cuestionable.

Coy analiza las relaciones entre la «frontera pionera» y el desarrollo urbano, estableciendo tipologías y diferencias socioeconómicas.

Un típico ejemplo de la descripción de algo exótico es el texto sobre el turismo tradicional en México de Tyrakowski, que ya escribía sobre el tema en 1986.

Uno de los últimos artículos de la revista Geographische Berichte es el de Breuler, que describe la estructura agraria latinoamericana, pero el texto aclara poco la situación y estructuras por el aparente desconocimiento de ésta por el autor.

El desarrollo y alguna que otra problemática conectada con él, son mencionados de alguna manera en casi todos los textos de este período. Aun así, pocas veces esto se relaciona con teorías sobre desarrollo. Tal vez se deba al poco conocimiento de los geógrafos de estas teorías, estudiadas principalmente por economistas. El carácter fuertemente descriptivo de muchos estudios sigue siendo la tónica general, aunque parece que la preocupación por explicar estructuras, problemáticas y procesos es mayor. Los espacios geográficos más analizados siguen siendo México y Brasil y seguidos de los Países Andinos.

También es interesante la total ausencia de artículos que relacionen desarrollo y medio ambiente, no sólo por ser un tema importante actualmente, sino también por ser un tema en el cual la Geografía podría jugar un papel importante.

Conclusiones sobre los articulos aparecidos en las revistas alemanas (1980-1994)

Un problema importante dentro de lo que la Geografía relacionada con Latinoamérica y la problemática del desarrollo en el ámbito alemán es que, muchas veces, se enfoca la problemática desde un

$$
-106-
$$


punto de vista muy tradicional. Es decir, que en vez de analizar procesos lo que se hace es una descripción de un lugar lejano y exótico y casi siempre a nivel local. Se describe o analiza un espacio con una problemática determinada, pero rara vez se llega más allá de esta descripción, rara vez se llega a hacer un diagnóstico o una propuesta para el futuro. Todo se plantea a escala local y rara vez se logra conectar diferentes escalas y dar una visión global del problema 0 , explicar las interdependencias existentes entre varias escalas. En cuanto a la base teórica relacionada con el desarrollo, es deficitaria en la mayoría de los artículos. Conceptos y teorías son apenas mencionados, y si lo son, se mencionan al margen y rara vez en conexión con el resto del texto.

En algunos textos parece que el autor tuviera poco conocimiento de la situación y realidad latinoamericana. Así, cuando algunos autores parecen esperar que las cosas funcionen o sean de la misma manera que en Europa, o cuando hacen descripciones en un tono paternalista, como si describieran una fiesta folklórica, se pone de manifiesto el eurocentrismo o mero desconocimiento de la realidad latinoamericana.

Muchos autores, aunque mencionan el concepto de centro-periferia y de la dependencia, sólo critican trabas endógenas al desarrollo, sin llegar a un nivel de análisis superior. Claro está que estas críticas no se reflejan en todo los textos, pero sí reflejan una imagen general de una temática que podría estar mucho más desarrollada dentro de la disciplina geográfica.

Las temáticas de los artículos tienen una evolución temporal. Mientras que en el primer período se centran en problemas agrarios, de construcción de viviendas y en los modelos urbanos, y aparece la primera crítica al «development from above», en el segundo período aparecen ya textos que tienen como cuestión principal la problemática social. Las críticas al «development from above» son más frecuentes y se habla del desarrollo rural integral. En este período aparecen también temas como la ecología y la identidad cultural, hasta entonces poco o nada problematizados.

En el tercer período se habla de conceptos como planificación endógena y exógena, y se presta más atención a medios y conceptos

$$
-107-
$$


locales de desarrollo, es decir, no importados desde Occidente. Se habla del autodesarrollo y del desarrollo local, de nuevo aparece la ecología y se menciona el uso sustentable de los recursos. También aparecen varios artículos que tratan del sector informal, un sector que dentro de lo que es la problemática del desarrollo va a seguir adquiriendo importancia en el futuro.

Se puede resaltar que hay una evolución positiva en el sentido de que parece haber más conciencia por parte de los autores y de que es necesaria la mención de la problemática del desarrollo en los textos, sobre todo en el último período.

\section{Referencias en las revistas estadounidenses}

En el período de 1980 a 1994, los principales asuntos tratados se pueden dividir en temas agrarios (Hiraoka-Yamamoto, 1989; Weil, 1983; Doolittle, 1983; Chardon, 1984), de industria y desarrollo (Cunningham, 1981; Chapman, 1982; Kipnis, 1983), urbano (GriffinFord, 1980), disparidades regionales (Haller, 1982; Hall, 1984) y turismo (Pearce, 1984).

Hiraoka-Yamamoto describen el desarrollo agrario en el «frente pionero» del Amazonas ecuatoriano en un análisis exhaustivo con diagnóstico y propuesta, desde un punto de vista liberal, pero que no llegan más allá del nivel local.

Weil estudia la migración de campesinos bolivianos en diferentes propiedades agrarias en un análisis también a nivel local, casi puntual. El autor concluye de forma general que la migración periódica supone una ventaja económica, pero no aclara el nivel socioeconómico de los afectados, ni los compara con otras poblaciones en situaciones similares, por lo cual, los resultados son escasos.

La expansión agraria en un área marginal de México en un nivel muy local y en forma descriptiva la analiza Doolittle.

El artículo de Chardon es un análisis de las plantaciones azucareras y la estructura de la propiedad en la República Dominicana, desde un punto de vista neoliberal y sin mencionar de manera alguna los problemas sociales existentes.

$$
-108-
$$


La equiparación de desarrollo con el desarrollo industrial es común en los tres textos de Cunningham, Chapman y Kipnis. Cunningham estudia dos ciudades medias brasileñas y sus estructuras industriales, trabajando con el concepto de «polo de crecimiento». Chapman escribe sobre las empresas multinacionales y sus patrones de localización en el Brasil, así como sus implicaciones para el desarrollo regional con resultados poco satisfactorios.

La industria petroquímica y el desarrollo son los temas de Kipnis en su artículo sobre Puerto Rico, en el que el autor presume que Puerto Rico se encuentra en una etapa de su desarrollo hacia lo que es el «ideal occidental».

La comparación de una Latinoamérica percibida como homogénea a anglo-américa es uno de los ejes principales del artículo de Griffin-Ford que plantean un modelo de la estructura urbana latinoamericana. El modelo se basa en un análisis exhaustivo de la situación y está bien planteado.

Dos estudios sobre disparidades regionales a nivel nacional entran en este período, uno de Haller sobre Brasil y otro de Hall sobre Costa Rica. Haller escribe con un enfoque cuantitativista, pero utiliza más variables que las puramente económicas. Hall establece los conceptos de "afluencia», como factor económico del bienestar, y bienestar social, que representa como las variables no directamente dependientes del mercado de capital privado. El autor hace una regionalización en base a estos factores.

El turismo en Belize, sus impactos positivos y negativos, son analizados por Pearce, que hace propuestas concretas para un planeamiento turístico futuro.

La mayoría de los textos están escritos con un enfoque que percibe el desarrollo, sobre todo, como algo económico, y desde un punto de vista neoliberal: la mano invisible del mercado soluciona todos los problemas. La mayoría de los textos son poco críticos y es notable la ausencia de teorías y conceptos sobre el desarrollo o de alternativas al enfoque liberal. Varios textos son de Geografía cuantitativa en la que se utilizan, exclusivamente, variables económicas y en otros se equipara desarrollo con desarrollo industrial, una idea que en otras 
ciencias se había dejado de difundir ya hace algún tiempo. Muchos autores tienden a comparar en demasía la situación latinoamericana con la anglo-americana.

A destacar, positivamente, es que la mayoría de los autores no sólo analizan una problemática, sino que hacen propuestas concretas para el futuro, que, casi siempre, es visto de forma positiva.

En el segundo período analizado, 1985 a 1989, encontramos pocos artículos: dos con temática agraria (Works, 1987; Lawson, 1988), dos sobre migración y desarrollo (Brown-Lawson, 1985; Lawson-Brown, 1987), uno sobre puertos y su desarrollo (Morti, 1985) y otro sobre desarrollo descentralizado (Greenow-Muñiz, 1988).

Works analiza la agricultura del pueblo indígena Aguaruna en Perú en un texto local y etnológico. Se dan aspectos interesantes en cuanto a los problemas ocasionados por un desarrollo occidentalizado, poco compatible con la cultura indígena.

Lawson hace un análisis cuantitativo examinando las interdependencias entre políticas interiores, producción agrícola y el cambio económico regional en Ecuador. La autora describe el desarrollo desequilibrado como una consecuencia directa de la política de industrialización por substitución de importaciones, que según ella se plantea en Ecuador en el año 1988. La primera es una argumentación cuestionable y la segunda una suposición igualmente cuestionable. El texto es crítico sobre todo en cuanto a las instituciones ecuatorianas y su funcionamiento, no llega más allá de la escala nacional, siendo sus resultados muy cuantitativos.

En el primer texto sobre migración y desarrollo, Brown-Lawson «descubren» que hay relaciones entre el nivel de desarrollo económico y las migraciones. En un artículo de características cuantitativas que trata del Tercer Mundo en general y de Costa Rica en especial. No se llega a considerar factores sociales dentro de lo que se denomina desarrollo. Los mismos autores, en su estudio sobre Venezuela llaman «tensión estructural» al desajuste entre la demanda y la oferta en el mercado de trabajo, causado por el crecimiento económico y la migración internacional. El texto es un análisis cuantitativo desde un punto de vista neoliberal, con datos estadísticos del año de 1971.

$$
-110-
$$


Morti analiza la especialización de puertos chilenos y sus potencialidades para la «containerización» y plantea un modelo de tres polos de crecimiento.

Greenow-Muñiz estudian el comercio en plazas de mercado en el Perú, el desarrollo descentralizado y el papel de la mujer (jprimer y último texto que incluye esta temática!). Llegan a la conclusión de que el comercio de mercado sólo, y difícilmente, estimula el desarrollo agrícola de sus «hinterlands».

El tercer y último período analizado, 1990 a 1994, lo caracterizan temas muy diferenciados. La «frontera pionera» desde diferentes puntos de vista (Godfrey, 1990; Roberts, 1992; Godfrey, 1992), temas urbanos (Godfrey, 1991), turismo y desarrollo sustentable (Zimmerer, 1991), desigualdades espaciles (Wall, 1993), organizaciones no gubernamentales (ONG's) y desarrollo (Price, 1994; Scarpaci, 1991), localización de industria (South, 1990), políticas espaciales (Klak, 1990), el sector informal (Hays-Mitchell, 1994) y agrarios (ZweiflerGold-Thomas, 1994).

Uno de los primeros autores estadounidenses de estas tres revistas que escribe sobre un desarrollo dependiente, orientado hacia el exterior, es Godfrey en su artículo sobre las ciudades «boom» en el «frente pionero» de la Amazonia brasileña. Estas ciudades tienen un crecimiento más rápido en el período en el cual la extracción de recursos minerales y madereros es la principal fuente de riqueza. Es un artículo que ofrece un buen análisis de la situación, pero que se queda algo corto en cuanto a propuestas.

En su segundo artículo sobre la «frontera pionera» Godfrey analiza la migración hacia una zona de minería del oro, las implicaciones sociales, así como la problemática medioambiental.

Roberts analiza el crecimiento urbano en una ciudad de la «frontera pionera». La ciudad que estudia está ligada, por localización, a un proyecto de desarrollo de gran envergadura. Roberts crea un modelo de invasión de tierras, causado por la falta de suelo urbano o urbanizable, por parte de «squatters» que se quieren asentar en la ciudad.

El turismo y el desarrollo sustentable en la sierra peruana son los temas expuestos del texto de Zimmerer. El texto es más de Geo- 
grafía Física, con muy pocas conexiones entre la escala local y otras mayores, sin apenas mención de la situación socioeconómica y de la problemática del desarrollo.

Una visión global de un país, Nicaragua, la da Wall. El autor analiza las desigualdades espaciales, comparando las metas de las políticas sandinistas en 1979 con los resultados hasta 1993. Aunque el autor reconoce que muchas de las metas no han podido ser realizadas por los costes de una guerra sustentada en gran parte por los Estados Unidos, el análisis no logra alejarse mucho de un punto de vista oficial estadounidense. No hace comparaciones con otros países, lo que sería útil para poder estimar el éxito o el fracaso de políticas que el autor, sin duda, considera fracasadas.

Un tema relativamente nuevo, pero cada vez más importante dentro de lo que es el desarrollo, son las ONG's. Éstas aparecen en el artículo de Price, que describe de forma global y general las ONG's medioambientales en Latinoamérica $y$ el cambio de modelos ecológico-proteccionistas hacia modelos del desarrollo sustentable. El artículo se centra, demasiado, en las relaciones de Estados Unidos con las ONG's para que pueda mostrar un cuadro no distorsionado.

Scarpaci estudia la conexión existente entre la descentralización de cuidados básicos, los movimientos sociales urbanos y el papel de las ONG's en el Cono Sur. Introduce el concepto del «estado local» y contrapone el desarrollo local y el poder central con intereses distintos. El artículo compara estos fenómenos en las capitales de Uruguay, Argentina y Chile y el cuidado de la saluden cada una de ellas.

Un texto interesante, porque se plantea la pregunta del desarrollo ¿para quién?, es el de South sobre la industria maquiladora y su localización en México y su fuerte conexión con los Estados Unidos, aunque la respuesta es superficial.

Un análisis bien logrado es el de Klak sobre programas y políticas estatales social y espacialmente progresivas en el Brasil. En este texto se compara la ejecución del programa habitacional del $\mathrm{BNH}$ (Banco Nacional da Habitaçao) en dos ciudades medias. Se llega a la conclusión de que las políticas son espacialmente progresivas (las zonas más desfavorecidas reciben más dinero), pero no socialmente 
progresivas (la gente más pobre, no es la más beneficiada). Un análisis y diagnóstico logrados que son seguidos de una propuesta de actuación clara:

La venta ambulante en el Perú y el sector informal son el tema de Hays-Mitchell en un análisis breve y localista.

Zweifler-Gold-Thomas analizan la evolución de los usos del suelo en la República Dominicana, comparando dos zonas muy específicas y puntuales. Pero aunque sea algo puntual, el análisis demuestra bien las interdependencias a varias escalas y niveles, plantea un modelo temporal y, también, de desarrollo local.

Los textos de estos dos períodos tienen temática muy variada, en el primer período tienden a ser cuantitativistas-economicistas, características que varían en el período 1990-1994. Los conceptos de desarrollo tienen una evolución bastante positiva, se llega a hablar de desarrollo dependiente y de desarrollo local. Temas nuevos importantes son las ONG's y el sector informal. La preocupación por dar pautas para el futuro y la planificación están bien planteadas y, sobre todo, en el último período, se introducen nuevos conceptos y modelos como son la planificación social y espacialmente progresiva, que podrán servir en estudios posteriores.

En los artículos de «frente pionero» se nota la total ausencia de los pueblos indígenas, y uno se pregunta si no son lo bastante geográficos o si es que simplemente no cuentan.

Conclusiones sobre los articulos aparecidos en las revistas estadounidenses (1980-1994)

Como en las revistas alemanas, en las estadounidenses se puede criticar que los textos se desarrollan sobre todo a una escala muy local y que son muy descriptivos, pero hay que recalcar que la mayoría de los autores estadounidenses hacen estos análisis incluyendo varias escalas $\mathrm{y}$, muchos, hacen propuestas para el futuro. Muchos autores parecen percibir a Latinoamérica como un espacio homogéneo y, demasiados, se basan en comparaciones con los Estados Unidos, lo que puede llevar a conclusiones no válidas. 
Si se tratan problemáticas del desarrollo, u otras estrechamente relacionadas, muchos autores ni siquiera sienten la necesidad de, por lo menos, indicar la situación socioeconómica de la población a que se

refieren. Muchos de los artículos que tratan directamente el tema de desarrollo son economicistas y de corte neoliberal, que, además de percibir el desarrollo sólo en términos económicos y, muchas veces, como una sucesión automática de etapas históricas en las que el libre mercado soluciona la mayoría de los problemas, sólo son capaces de discernir causas endógenas de subdesarrollo. Este enfoque se pone en evidencia en los artículos cuantitativistas, al comienzo de los ochenta, y se evoluciona después hacia una visión mucho más pluralista al comienzo de los noventa. Así, el primer texto que menciona el desarrollo dependiente aparece en el tercer período, donde también se menciona el desarrollo sustentable y se relaciona desarrollo y medio ambiente.

En el tercer período tienen más peso los temas sociales. Se tratan los movimientos sociales urbanos, el papel de la mujer en el desarrollo y se plantea la pregunta: ¿desarrollo para quién?

\section{Análisis comparado: revistas alemanas-revistas estadounidenses}

Aunque dentro de los textos de las revistas de los dos países existen muchas similitudes, como son el predominio de las temáticas agrarias y urbanas, muchas veces conectadas a lo que se denomina «frente pionero» o el turismo, es notable que la variedad temática en las revistas estadounidenses es mayor. En ambos países ha habido una apertura hacia un temario más variado con el tiempo, sobre todo en los últimos cinco años. Aunque muchas veces esta apertura es positiva, otras se plantean la pregunta de que si algunos textos pueden o deben llamarse geográficos.

En la Geografía alemana parece haber una tendencia a hacer estudios más tradicionales, siendo la descripción una parte importante, mientras que los estadounidenses se prestan más al análisis interescalas y a conectar análisis, diagnóstico y propuesta.

En cuanto al enfoque que tienen los autores sobre desarrollo, en los dos ámbitos destaca que la problemática del desarrollo sólo merece una mención marginal, como si fuera un problema secundario. 
Los textos norteamericanos, en su mayoría, tienen un enfoque neoliberal, con la convicción de que el mercado lo solucionará todo si le dejamos actuar, es decir, si eliminamos las trabas a nivel espacial, cultura, social y estatal. La idea de un desarrollo en etapas o fases de sucesión automática también está muy poco tratada y la preocupación por el medio ambiente o factores culturales no se puede llamar extremadamente fuerte. Estas posiciones neoliberales están un poco atenuadas en el último período. Los autores americanos utilizan teorías espaciales, sobre todo en la rama cuantitativa, y son mucho más proclives a tener una imagen positiva de lo que puede llegar a ser el futuro, mientras que los alemanes son más escépticos.

En las revistas alemanas muchas veces es aún más difícil entrever qué concepción del desarrollo tiene el autor. Se puede considerar que también gran parte mantiene una postura liberal. Los conceptos como la dependencia y el centro-periferia ya se pueden encontrar en los primeros textos. Son en general más críticos y más descriptivos y algunos tienen posturas negativas hacia lo que se podría denominar el desarrollo occidental.

La percepción de Latinoamérica en ambos países es, como era de esperar, relativamente distante, aunque también depende mucho del conocimiento de cada autor.

Los autores norteamericanos tienden a tener una imagen menos diferenciada de lo que es Latinoamérica que los europeos, lo que sorprende, ya que por el mayor contacto cultural de los estadounidenses con los latinoamericanos y su cercanía geográfica se podría esperar que la imagen fuera otra. Los europeos a su vez tienden a ser más paternalistas que los estadounidenses, éstos tienden a comparar la realidad estadounidense con la latinoamericana y a transcribir sus teorías fundadas en experiencias estadounidenses al contexto latinoamericano: «Las discusiones de la teoría regional y urbana, miradas desde cerca, están basadas totalmente en los datos y perspectivas de América del Norte (...). No obstante, los geógrafos norteamericanos siguen muy dispuestos a ofrecer sus percepciones teóricas derivadas de las praderas de Iowa, de los ghettos de Chicago, de los suburbios de Boston o de las experiencias metropolitanas de Los Ángeles» (Robinson-Long, 1989, p. 304). Aunque lo que re- 
marcan los autores en cuanto a los geógrafos norteamericanos no es válida sólo para éstos cuando hablan: «del notable etnocentrismo de nuestra disciplina» (Robinson-Long, 1989, p. 304).

\section{Conclusiones}

¿Ha habido un progreso dentro de la Geografía en lo concerniente a la problemática del desarrollo y Latinoamérica? Sin duda la respuesta para los últimos quince años es sí. Aunque existan muchos puntos críticos o criticables, hay que reconocer que ha habido una evolución positiva, a pesar de que quedan muchas lagunas y muchos aspectos mejorables.

La conciencia de que al hablar sobre Latinoamérica y sus estructuras espaciales, hay que mencionar de alguna manera la problemática del desarrollo se ha agudizado. En los últimos años se ha empezado a trabajar con conceptos del desarrollo local y endógeno, que parte de ideas, conceptos y potenciales locales y que es ejecutado en parte importante por las personas directamente afectadas.

Constantemente en estos quince años se han incluido nuevos temas. La conciencia de que hay una identidad cultural y un ambiente que se debería conservar dentro de lo que se denomina desarrollo sostenible se va haciendo mayor, aunque, hasta ahora, de forma algo leve. También se está incluyendo a nuevos actores dentro de lo que es el desarrollo, como las ONG's o el sector informal.

En cuanto a los conceptos de desarrollo y las teorías que lo sustenta, también se puede ver cierto progreso. Mientras que en los ochenta dominan ideas económicas neoliberales, de lo que se llamó la contrarrevolución del pensamiento de desarrollo: «Los partidarios de una contrarrevolución en teoría y política del desarrollo están acostumbrados a describir la economía política global como un macroespacio interdependiente tendente al equilibrio y balance espacial en virtud del funcionamiento ininterrumpido del capital internacional, el comercio y los mercados monetarios y de trabajo» (Corbridge, 1990 , p. 392). En los noventa, esta concepción ya no es la única válida. Muchos autores aún defienden estrategias neoliberales, los pun-

$$
-116-
$$


tos de vista se han ampliado, y se puede encontrar autores que defienden otros puntos de vista y se atreven a preguntar ¿desarrollo para quién? (South, 1990).

Pero como ya se ha dicho, aún falta mucho camino por recorrer, como pone de manifiesto un texto de Ridell sobre la Geografía y los estudios del subdesarrollo y del Tercer Mundo, al preguntarse él por qué no aparecen geógrafos en importantes publicaciones con temas sobre desarrollo: «¿Es la respuesta un problema únicamente de escala: que nuestro trabajo tiende a ser de nivel micro mientras que el interés de otras muchas disciplinas es nacional? o ¿es la causa del legado físico determinista de la Geografía o de la descripción enciclopédica de regiones únicas lo que hace que nuestro tema sea menos trascendente?» (Ridell, 1987, p. 264).

Además de éstas, cabe hacer otras preguntas críticas como: ¿Ha cambiado la percepción de Latinoamérica por parte de los autores con el tiempo, o sigue habiendo muchos que en sus textos parece que hicieran una descripción folklorista o que escriben con un tono paternalista? ¿Sigue siendo la mayoría de los textos euro o anglo-americanos centristas?

¿Se sigue desatendiendo ciertos temas?, como la de los pueblos indígenas, y ¿la distribución de artículos sigue siendo tan polarizada espacialmente?

Hay todavía muchos autores que mencionan la problemática del desarrollo sólo como problema marginal, que no mencionan las causas del subdesarrollo o que sólo llegan a criticar trabas endógenas al desarrolo, sin ver las interdependencias y dependencias globales.

Seguramente la mayoría de las preguntas pueden ser respondidas afirmativamente, como también observa Ridell: «Lo primero es que la investigación geográfica tiende a ser microespacial (...), sin embargo, al mismo tiempo, las disciplinas relacionadas con los estudios de desarrollo se han preocupado generalmente por el nivel macroespacial tanto nacional como internacional. Segundo, ha habido una continuación de la tradición "de ingeniería espacial" del análisis geográfico en el Tercer Mundo. Esta posición indica especialmente que una de las causas importantes de la dificultad del Tercer Mundo es que el espa- 
cio (o Geografía) está estructurado inadecuadamente. En efecto, se está sugiriendo que si la organización espacial puede ajustarse adecuadamente, será posible que tenga lugar el desarrollo. Por eso esta literatura sugiere que las barreras al avance de los países del Tercer Mundo sólo son internas en aquellas áreas -una noción ignorada para gran parte del progreso teórico y empírico de las dos últimas décadas» (Ridell, 1987, p. 272).

También es notable la falta de modelos y teorías propiamente geográficas, aunque esto no sólo es un problema en la rama de la Geografía que trabaja con la problemática de desarrollo. Aun así es necesario avanzar en esta dirección, o sea, en la creación de teorías propias, ya sea por el bien de la disciplina o ya sea por que el campo de estudio no es sólo geográfico. Algunos argumentan que el campo es muy poco geográfico, en el sentido de que pocos geógrafos trabajan en él. Según Gilbert eso no se debe a que falten temas interesantes que estudiar: "Ciertamente esto no se debe a la ausencia de temas de interés que reclaman análisis (...). A veces es verdad que los sociólogos y los antropólogos han colonizado lo que podría reivindicarse como espacio geográfico. Pero uno sospecha que los geógrafos no habían trabajado en estos temas» (Gilbert, 1986, p. 542).

Pues si esto es así, no queda más que convencer a más geógrafos de que se ocupen del tema y que lo hagan seriamente. ¿Y qué es en lo que estos geógrafos deberían concretarse? ¿Qué es lo que hay que hacer para lograr un progreso? La pregunta es difícil de responder, aunque parte de la respuesta seguramente está en lo que dice Ridell: «... la Geografía como esclava de la historia». Para evitar tal desconcierto debemos cambiar desde la descripción del modelo al estudio del proceso que afecta y causa tales expresiones zonales» (Ridell, 1988, p. 112).

Es decir, caminar hacia una ciencia menos estática, de una ciencia de descripción de lugares exóticos a una ciencia activa, que además de analizar los procesos que causan las estructuras espaciales (o son causados por éstas), los geógrafos lleguen a diagnosticar y a hacer proposiciones válidas para el futuro, aun cuando resulte difícil.

En los tiempos cuando la brecha entre el «norte» y el «sur» se hace más grande y en los que la problemática medioambiental y so-

$$
-118-
$$


cial es cada vez más acusada, hay muchos campos y muchos problemas que quedan por explicar $\mathrm{y}$, sobre todo, por resolver. Y los geógrafos seguramente tienen técnicas y conocimientos válidos para responder a algunas de estas custiones, tanto en lo referente a Latinoamérica como a la problemática del desarrollo. Como dice Corbridge (1992), buscando el equilibrio entre el activismo local y la sensibilidad hacia las injusticias globales.

\section{BIBLIOGRAFÍA}

Revistas consultadas

Annals of the Association of American Geographers, del núm. 1 de 1980 (AAAG, vol. 70) al núm. 1 de 1995 (AAAG, vol. 85).

Die Erde, del núm. 1 de 1980 (DE, Jahrgang, 111) al núm. 1 de 1995 (DE, Jahrgang, 126).

Erdkunde, del núm. 1 de 1980 (ERDK, Band, 34) al núm. 4 de 1986 (ERDK, Band, 40) y del núm. 1 de 1989 (ERDK, Band, 43) al núm. 1 de 1995 (ERDK, Band, 49.

Geographical Review, del núm. 1 de 1980 (GR, vol. 70) al núm. 4 de 1984 (GR, vol. 74) y del núm. 1 de 1986 (GR, vol. 76) al núm. 1 de 1994 (GR, vol. 84).

Geographische Berichte, del núm. 1 de 1980 (GB, núm. 98) al núm. 3 de 1990 (GB, núm. 136).

Geographische Zeitschrift, del núm. 1 de 1980 (GZ, Jahrgang, 68) al núm. 3 de 1994 (GZ, Jahrgang, 82).

Petermanns Geographische Mitteilungen, del núm. 1 de 1980 (PGM, núm. 1/80) al núm. 4 de 1994 (PGM, núm. 4/94).

The Professional Geographer, del núm. 1 de 1980 (PG, vol. 32) al núm. 2 de 1995 (PG, vol. 47).

Artículos y libros (sólo aparecen los artículos que han sido importantes en la elaboración del texto o aparecen en él):

BÄHR, J., y Mertins, G. (1981): «Idealschema der sozialräumlichen Differenzierung lateinamerikanischer Grossstädte», GZ, 69, pp. 1-33.

- (1985): «Bevölkerungsentwicklung in Gross-Santiago zwischen 1970 und 1982. Eine Analyse von Zensusergebnissen auf Distriktbasis», ERDK, pp. 218-238.

BIRK, F. (1993): «Indianische Kleinbauern im Hochland Guatemalas, ihre Alltagslogik und interne Organisation", $G Z, 81$.

BlECKERT, H. (1983): «Aktuelle Aspekte der Auseinandersetzung zwischen den Andenblockländern und dem imperalistischen Monopolkapital», $P G M, 1 / 83$.

Blomströn, M., y Hettne, B. (1984): La teoría del desarrollo en transición, Fondo de Cultura Económica, México, 1990. 
BReUler, S. (1990): «Die Agrarstuktur Lateinamerikas im Überblick», GB, pp. 39-52.

Brogner, D. (1984): «Metropolisierung als Entwicklungsproblem in den Ländern der Dritten Welt. Ein Beitrag zur Begriffsbestimmung», GZ, 72, pp. 138-158.

Brown, L. A., y Lawson, V. A. (1985): «Migration in Third World settings, uneven development, and conventional modeling: a case study of Costa Rica», $A A A G$, 75, pp. 2947.

BUCHHOFER, E. (1984): «Minatitlán (Mexiko) - zur Sozialtopographie einer company town in der Dritten Welt», GZ, 72, pp. 159-178.

- (1986): «Resultate öffentlicher Bodenpolitik un Wohnungsbauförderung in jungen mexikanischen Industriestädten am Pazifik», DE, 117, pp. 237-257.

- (1988): «Wirtschaftsgeographische Grundlagen der Stadtentwicklung im ecuadorianischen Amazonas-Tiefland», GZ, 76, pp. 149-164.

Buchiofer, E., y Guillermo Aguilar, A. (1991): «Stadtexpansion in der Schuldenkrise. Der fall Mexiko-Stadt», GZ, 79, pp. 26-43.

Chapman, K. (1982): «Petrochemicals and Economic Development. The Implications of the Puerto Rican Experience», PG, 34, pp. 405-415.

Chardon, R. (1984): «Sugar plantations in the Dominican Republi», GR, 74, pp. 441-454.

CoRBRIDGE, S. (1986): «Capitalism, industrilization and development», $P G, 10$, pp. 4867.

- (1990): «Development studies», $P G, 14$, pp. 391-403.

- (1991): «Third world development», $P G, 15$, pp. 311-321.

- (1992): «Third world development», $P G, 16$, pp. 584-595.

Cotic, B. A., y Zothner, V. (1988): «Historisch-geographische Entwicklung von Buenos Aires», GB, 126, pp. 51-62.

Coy, M. (1986): «Regionalentwicklung in Rondônia (Brasilien). Integrierte ländliche Entwicklung und politische Rahmenbedingungen», GZ, 74, pp. 177-185.

- (1990): «Pionierfront und Stadtentwicklung. Sozial - un wirtschaftasräumliche Differenzierung der Pioneirstädte in Nord-Mato Grosso (Brasilien)», GZ, 78, pp. 115-134.

Cunningham, S. M. (1981): «Multinational Enterprises in Brazil: Locatinal Patterns and Implications for Regional Development», $P G, 33$, pp. 48-62.

Doolittle, W. E. (1983): «Agricultural expansion in a marginal area of Mexico», GR, 73, pp. 301-313.

Friebel, W. U. (1983): «Untersuchungen zur Agrarstruktur indianischer Gemeinden in der chilenischen Araucania (IX Región)», ERDK, 37, pp. 47-59.

GANS, P. (1990): «Wirtschafliche Entwicklung und informeller Sektor in Lateinamerika. Das Beispiel des ambulanten Handels in Montevideo», GZ, 78, pp. 48-62.

GIERHAKe, K. (1991): «Die Rolle der öffentlichen Investitionen im Prozess der Regional entwicklung in Bolivien (1987-1990)», GZ, 79, pp. 229-245.

- (1993): «Regionale Entwicklungsplannung in marginalisierten Räumen des andinen Lateinamerika. Beispiele aus Bolivien, Kolumbien, Peru», DE, 124, pp. 37-51.

GILBERT, A. (1986): «Latin American studies», PG, 10, pp. 541-552.

Godfrey, B. J. (1990): «Boomtowns of the Amazon», GR, 80, pp. 103-117.

- (1991): «Modernizing the brazilian city», GR, 81, pp. 18-34.

- (1992): «Migration to the gold-mining frontier in brazilian amazonia», GR, 82, pp. 458-469. 
GoRMSEN, E. (1981): «Die Städte im spanischen Amerika - Ein zeit-räumliches Entwicklungsmodell der letzten hundert Jahre», ERDK, 35, pp. 390-302.

- (1983): «Der internationale Tourismus, eine neue "Pionierfront" in Ländern der Dritten Welt», GZ, 71, pp. 149-166.

- (1986): «Der Flughafen als Standortfaktor für den Blumenanbau am Beispiel von Kolumbien», ERDK, 40, pp. 305-317.

Greenow, L., y MuÑIz, V. (1988): «Market Trade in Decentralized Development: The Case of Cajamarca, Peru», $P G, 40$, pp. 416-427.

Griffin, E., y Ford, L. (1980): «A Model of Latinamerican City Structure», GR, 70, pp. 397-422.

Gristal, O., y Treivish, A. (1990): «Stadial Concept of Regional Development: The Dynamics of Core and Periphery. A theoretical Discussion», GZ, 78, pp. 65-78.

HaLl, C. (1984): «Regional inequialities in well-being in Costa Rica», GR, 74, pp. 48-62.

HALLER, A. O. (1982): «A socioeconomic regionalization of Brazil», GR, 72, pp. 450-464.

Hays-Mrtchel, M. (1994): «Streetvending in Peruvian Cities. The Spatio-Temporal Behaviour of Ambulante», $P G, 46$, pp. 425-438.

HeineberG, H., y Schäfers, C. (1989): «Metropolisierung und Probleme der Raumplanung in Mexiko, Fallstudie Matropolitangebiet von Guadalajara», $D E$, 120, pp. 99-119.

Hiraока, M., у Yамамото, S. (1980): «Agricultural development in the upper amazon of Ecuador», GR, 70, pp. 423-445.

Hönsch, F. (1986): «Herausbildung, Probleme und Tendenzen der Zuckerwirtschaft Kubas», GB, 119, pp. 107-123.

JURCZEK, P. (1985): «Gross- und kleinräumige auswirkungen des Ferntourismus auf Peru», $D E, 116$, pp. 27-47.

KIPNIS, B. A. (1983): «Clusters and Concepts of Medium-sized Urban Manufacturing Systems: Two Case Studies in Brazil», PG, 35, pp. 32-39.

KLAK, T. (1990): «Spatially and Socially Progressive State Policy and Programs: The case of Brazil's National Housing Bank», $A A A G$, 80, pp. 571-589.

KoHLHEPP, G. (1989): «Struckturwandlungen in der Landwirtschaft und Mobilität der ländlichen Bevölkerung in Nord-Paraná (Südbrasilien)», GZ, 77, pp. 42-61.

KoRPE, B. (1986): «Das agrare Genossenschaftswesen als raumgestaltender Faktor. Zur Ambivalenz gelenkter Modernisierung am Beispiel von West-Paraná (Brasilien)», GZ, pp. 241-249.

Lawson, V. (1988): «Government Policy Biases and ecuadorian agricultural change», $A A A G, 78$, pp. $433-452$.

-, y Brown, L. A. (1987): «Structural Tension, Migrations and Development: A Case Study of Venezuela», $P G, 39$, pp. 179-188.

LÜCKER, R. (1986): «Räumlicher Struckturwandel in einer peripheren Region durch weltmarktorientierte Agrarpolitik. Das Beispiel Alto-Uruguai (Südbrasilien)», GZ, 74, pp. 168-176.

MaHnKe, L. (1982): «Zur indianischen Landwirtschaft im Siedlungsgebiet der Kallawayas (Bolivien)», ERDK, 36, pp. 247-253.

Mikes, W. (1983): «Die grundbedürfnisorientierte Regionalanalyse - Schwächen der Arbeitsmarktstruktur Lateinamerikas unter besonderer Berücksichtigung von Peru», GZ, 71, pp. 87-106. 
Morti, B. E. (1985): «Chilean Ports: Comodity Specialization and Potential for Containerisation», $P G, 37$, pp. 320-328.

NuHN, H. (1981): «Struktur und Entwicklung des Städtesystems in den Kleinstaaten Zentralamerikas und ihre Bedeutung für den regionalen Entwicklungsprozess», ERDK, 35, pp. 303-320.

Pearce, D. G. (1984): «Planning for tourism in Belize», GR, 74, pp. 291-303.

PoPP, K. (1985): «Privatwirtschaftliche Baulanderschliessungen (fraccionamientos) im Zuge der Stadterweiterung mexikanischer städte aufgezeigt am Beispiel der Stadt Puebla/Pue», ERDK, 39, pp. 144-152.

PRICE, M. (1994): «Ecopolitics and environmental non-governmental organizations in Latin America», GR, 84, pp. 42-58.

REHKER, J. R. (1989): «Untersuchungen zum Rückgang der kleinbäuerlichen Grundnahrungsmittelproduktion in ausgewählten Munizipien des nordostbrasilianischen Agreste», ERDK, 43, pp. 119-132.

RIDELL, J. B. (1987): «Geography and the study of third world undervelopment», $P G$, 11, pp. 264-274.

- (1988): «Geography and the study of third world underdevelopment revisted», $P G$, 12, pp. 111-120.

- (1989): "Geography and the study of third world underdevelopment re-revisted», $P G, 13$, pp. 267-276.

RoBERTS, J. T. (1992): «Squatters and urban growth in Amazonia», GR, 82, pp. 441-457.

RoBINson, D. J., y LoNG, B. K. (1989): «Trends in latin americanist geography in the United States and Canada», PG, 41, pp. 304-314.

Rönick, V. (1983): «Ländliche Regionalentwicklung in Nordost-Brasilien: Das Beispiel der "Serra da Ibiapaba“", GZ, 71, pp. 50-61.

Sagawe, T. (1989): «Drogenphänomene als Stadtgeographische Fragestellung am Beispiel von Santo Domingo», GZ, 77.

- (1992): «Caudillismo und Stadtentwicklung. Das Beispiel von Santo Domingo», GZ, 80 , pp. 51-64.

SANDER, H.-J. (1981): «Beziehungen zwischen Tourismus, ländlichem Kunsthandwerk und Agrarstruktur in einigen Dörfern Zentralmexiko», ERDK, 35, pp. 201-209.

SCARPACI, J. H. (1991): «Primary-Care Decentralization in the Southern Cone: Shantytown Health Care as Urban Social Movement», AAAG, 81, pp. 103-126.

SсHACHт, S. (1980): «Agrarkolonisation in der Zona da Mata Nordostbreasiliens am Beispiel der Kolonie Pindorama», GZ, 68, pp. 54-76.

SchneIder-Sliwa, R. (1991): «Exogenous and Endogenous Concepts of Urban Planning: Urban Revitalization in Kingston, Jamaica», $D E$, 122, pp. 3-22.

SeELE, E. (1986): «Strukturwandlungen in der Agrarwirtschaft im Tal von Tehuacán/ Pue.-México», ERDK, 40, pp. 283-293.

South, R. B. (1990): «Transnational "Maquiladora" Location», AAAG, 80, pp. 549-570.

Todaro, M. P. (1985): El desarrollo económico del Tercer Mundo, Alianza Editorial, Madrid, 1988.

TYrakowski, K. (1986): «Zur Entwicklung eines einheimischen Fremdenverkehrs in Mexiko, dargestellt am Beispiel des Staates Tlaxcala», ERDK, 40, pp. 293-304.

- (1994): «Wallfahrer in Mexiko - eine Form traditionelle Fremdenverkehrs zwischen "kultischer Ökonomie" un Tourismus», ERDK, 48, pp. 60-74. 
UNGEFEHR, F. (1988): «Freeport/Gran Bahama - ein privates Entwicklungsprojekt in den Netzen der bahamanischen Innenpolitik», $D E, 119$, pp. 99-110.

WACHTER, P. (1992): «Die Bedeutung des Landtitel-besitzes für eine nachhaltige landwirtschaftliche Bodennutuzung. Eine empirische Fallstudie in Honduras», GZ, 80, pp. 174-183.

WALL, D. L. (1993): «Spatial inequalities in sandinista Nicaragua», GR, 83, pp. 1-13.

WATTS, M. J. (1993): «Development 1: power, knowledge, discursive practice», $P G, 17$, pp. 257-272.

WeHRHAhn, R. (1993): «Ökologische Probleme in lateinamerikanischen Grossstädten», $P G M$, núm. 2/93, pp. 79-94.

WeILL, C. (1983): «Migration among landholdings by bolivian campesinos», GR, 73, pp. 182-197.

Works, M. A. (1987): «Aguaruna agriculture in eastern Peru», GR, 77, pp. 349-362.

ZimmereR, K. S. (1991): «Tourism and sustainable development in the southern peruvian sierra», GR, 81, pp. 414-432.

Zweifler, M. O.; GOLD, M. A., y Thomas, R. N. (1994): «Land Use Evolution in Hill Regions of the Dominican Republic», $P G, 46$, pp. 39-53.

RESUMEN: El artículo pretende dar una noción de lo que ha sido en los últimos años el estudio de la problemática del desarrollo en los países latinoamericanos dentro de la geografía alemana y estadounidense, a través de un estudio bibliográfico sobre este tema realizado en revistas geográficas de estos países y publicadas en los últimos quince años (1980-1994).

Palabras Claves: Latinoamérica. Desarrollo. Revisión bibliográfica.

ABstract: This paper tries to give a notion of what is has been during the last years, the development study of the set of problems in the Latin American countries on the inside of the Germany Geografy and United States, trough a bibliographic study on the topic, made in geographys magazines in these countries and published during the last fifteen years (1980-1994).

Keywors: Latin America. Development Bibliographic review. 